\title{
Routing of Fluidic Circuits Using Skeletons of Polygonal Figures and Compound Splines
}

\author{
Vladimir Romakin ${ }^{1, *}$ \\ ${ }^{1}$ V.A.Trapeznikov Institute of Control Sciences of Russian Academy of Sciences, Moscow, Russia
}

\begin{abstract}
Routing of channels between elementary fluidic elements is an important stage in the design of new fluidic devices. This paper considers usage skeletons of polygonal figures and compound splines in routing of fluidic circuits.
\end{abstract}

\section{Introduction}

Routing of channels between elementary fluidic elements is an important stage in the design of new fluidic devices. The geometry of working channels of fluidic devices significantly affects their performance [1]. The communication channels between the fluid elements must have a given width, which can vary along the channel, and should be sufficiently smooth. Sharp turns and kinks can lead to a significant deterioration in the characteristics of the product, as they cause additional resistance to the flow of gas or liquid, the occurrence of turbulence and a significant change in flow dynamics. The routing algorithms, used in the design of printed circuit boards do not allow the creation of the required connections between fluidic elements.

In the previous work [2] a method was proposed for constructing a topological model of channels of fluidic devices using skeletons of polygonal figures. This paper considers usage compound splines in constructing geometric models of channels of fluidic devices.

\section{Constructing a topological model of channels}

The initial data are two-dimensional geometric models of fluidic elements, the coordinates of their placement on the board, as well as the functional relationships between the inputs and outputs of the fluidic elements. See Figure 1.

The topological model of the channels of the fluidic device is constructed as follows. First, a polygonal figure is constructed, in which the outer border describes the boundary of the board of the fluidic device, and the "holes" are the contours of the fluidic elements placed on this board. Then a straight skeleton of the polygonal figure and a planar graph corresponding to it are constructed. Finally, sequentially for each functional connection, a path on the graph is found that connects the output of one element with the input of another element [2]. Figure 2 shows the outline of the polygonal figure in black, the straight skeleton in red and green, and the paths found on the graph in yellow.

\footnotetext{
* Corresponding author: insight.ru@gmail.com
} 


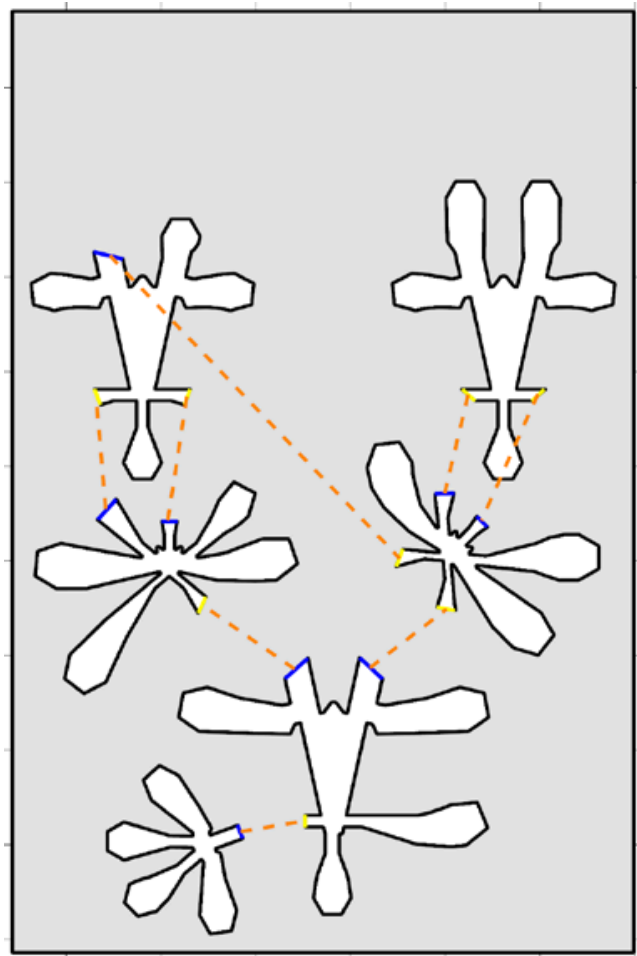

Fig. 1. An example of a model of a fluidic device with functional connections

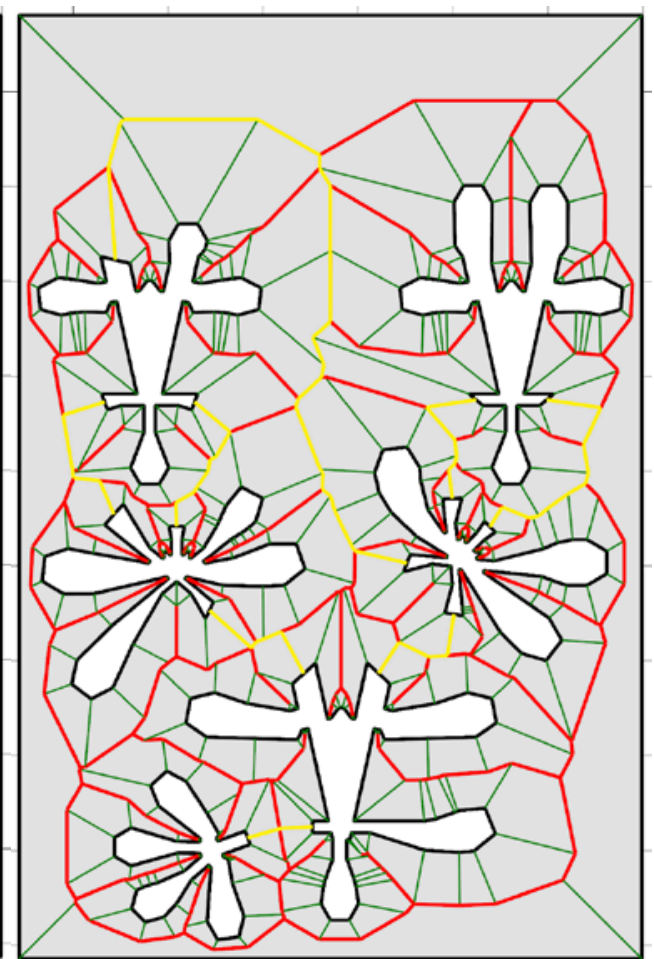

Fig. 2. Polygonal figure of the model and its straight skeleton with selected traces

The polygonal chains (polylines) representing the found paths on the graph are characterized by a large number of kinks, therefore, they are considered only as a topological model of the channels. The problem of constructing the geometry of the channels can be solved by smoothing these polylines.

\section{Building a geometric model of channels}

To smooth the polyline, an iterative method for constructing a smooth spline consisting of cubic Bezier curves and approximating a given polyline with the required accuracy has been developed.

Let's set the number of spline interpolation nodes equal to $N$ and place the interpolation nodes evenly along the polyline, so that the two extreme nodes lie on the start and end points of the polyline, and the lengths of the polyline segments between neighboring nodes are equal. Let's construct a smooth spline passing through the given interpolation nodes. To do this, we will use the well-known method of constructing a smooth spline consisting of $\mathrm{N}-1$ cubic Bezier curves and passing through given points (interpolation nodes) [3]. The initial data in this method are interpolation nodes, and the coordinates of the control points of the Bezier curves are calculated using a system of $2 \mathrm{~N}-2$ linear equations that ensure the continuity of the first and second derivatives of the spline at the interpolation nodes. For each Bezier curve $B_{i}(i=1, . ., N-1)$ of the constructed spline, we calculate the approximate area $S_{i}$ of the region between curve boundary and the segment of the polyline between the two interpolation nodes of the curve. The area values will be used, on the one hand, to evaluate accuracy, with which the constructed spline approximates the polyline, and on the 
other hand, to move interpolation nodes. If the sum of the areas $S_{1}+\ldots+S_{N-1}$, that is, the area of the region between the constructed spline and the polyline, exceeds the allowable value, then we will consider the approximation accuracy insufficient and try to improve it. For each intermediate $i$-th interpolation node $(i=2, . ., N-1)$, we compare the areas calculated for two adjacent Bezier curves $S_{i-1}$ and $S_{i}$, and move the interpolation node along the polyline towards the region with a large area value. The distance to which the interpolation node is moved will be selected taking into account the distance to the adjacent interpolation node, towards which the movement is performed, and a larger area value. After moving all intermediate nodes, again construct a smooth spline passing through them, and estimate the accuracy of the approximation. We will iterate until the required approximation accuracy is achieved. In addition to the approximation accuracy, we will additionally check that the constructed spline is free of self-intersections, does not approach the boundaries of the fluidic elements closer than half the channel width, and its curvature does not exceed the maximum allowable value. If it was not possible to achieve the required conditions in a given number of iterations, we increase the number of interpolation nodes $N$ and repeat the whole procedure again, starting with the uniform placement of interpolation nodes along the polyline.

Let us consider the proposed method using the example of the approximation of the polyline shown in Fig. 3. Let us fix $N=5$. Place five interpolation nodes evenly along the polyline and construct a smooth spline passing through these nodes and consisting of four Bezier curves.

Figure 4 shows the interpolation nodes and the spline line in brown, and the regions between the Bezier curves and the corresponding sections of the polyline in red, orange, yellow and green. Let's calculate the areas of these regions. Since the sum of the areas is large enough, let's move three intermediate interpolation nodes, each towards an adjacent region with a larger area. Figure 5 shows the spline obtained after first moving intermediate nodes. We will calculate the areas of the regions and move the interpolation nodes (Fig. 6, 7) until the sum of the areas becomes small enough (Fig. 8).

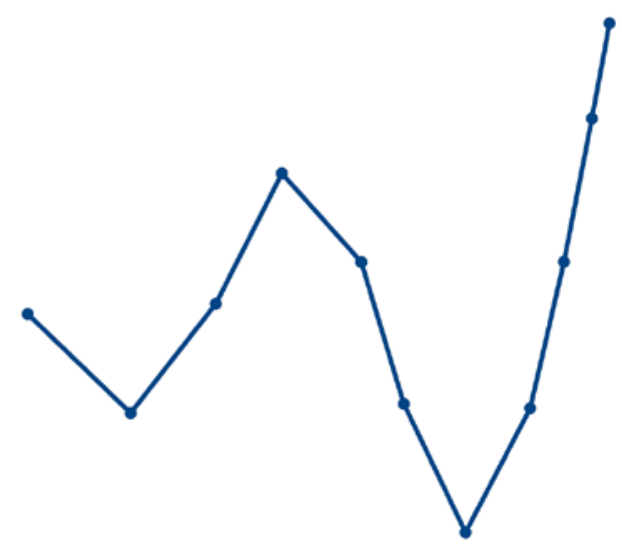

Fig. 3. Example of polyline to approximate

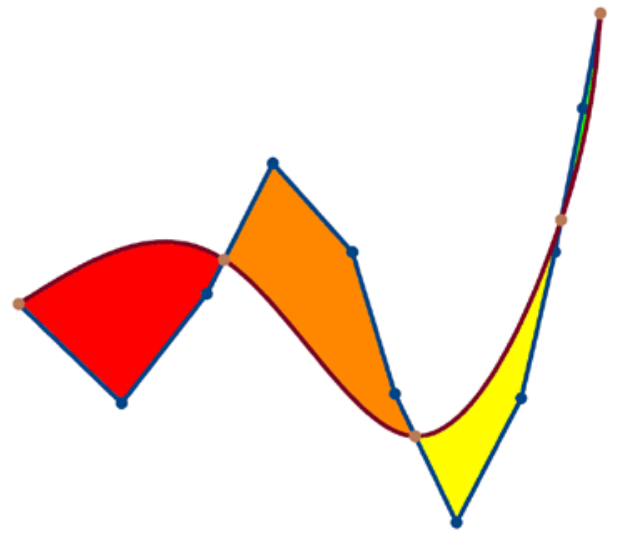

Fig. 4. Spline at the first iteration 


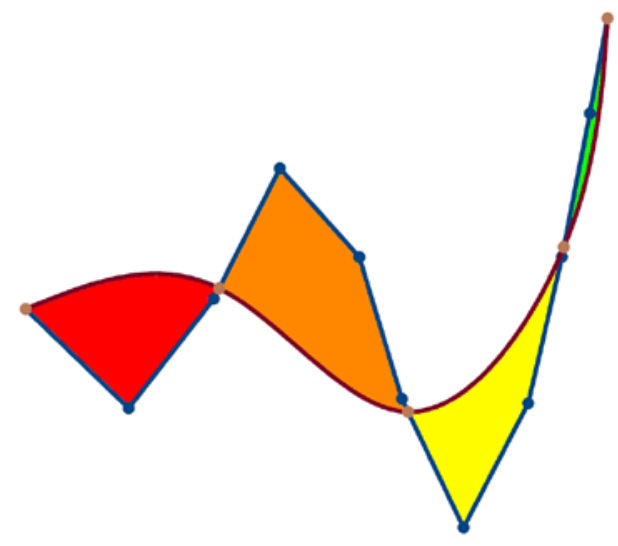

Fig. 5. Spline at the second iteration

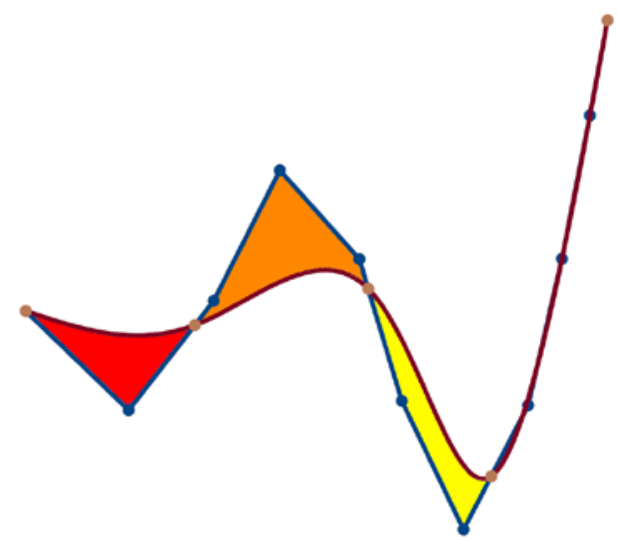

Fig. 7. Spline at one of the next iterations

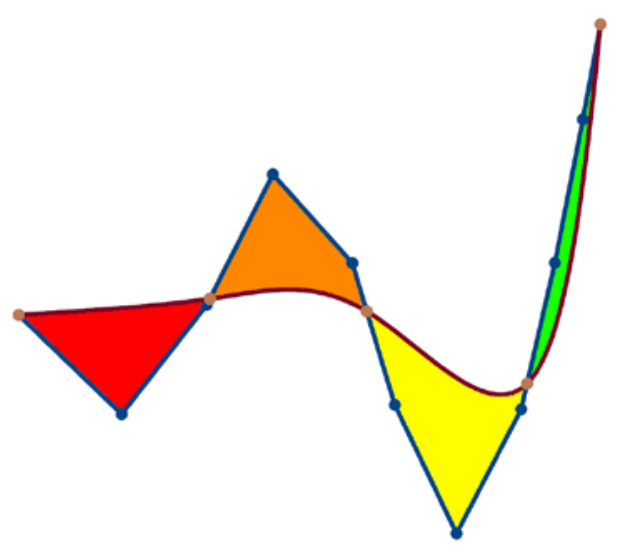

Fig. 6. Spline at one of the next iterations

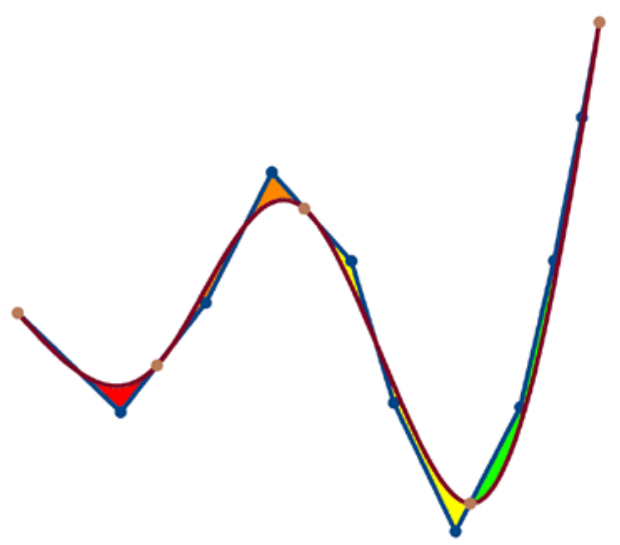

Fig. 8. The resulting spline

Let's return to the task of constructing a geometric model of channels. In fig. 9 and 10 blue color shows a polyline connecting the output of one fluidic element with the input of another element, and brown color shows the resulting spline obtained by the proposed method at different values of $N$. It can be seen that at $N=4$ the approximation accuracy is very low (Fig. 9), and at $N=5$ it is already quite acceptable (Fig. 10). 


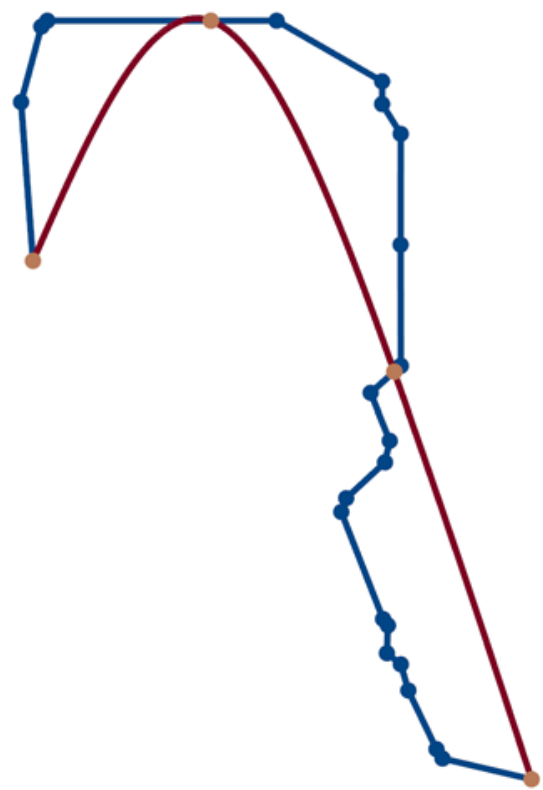

Fig. 9. Spline example with four nodes

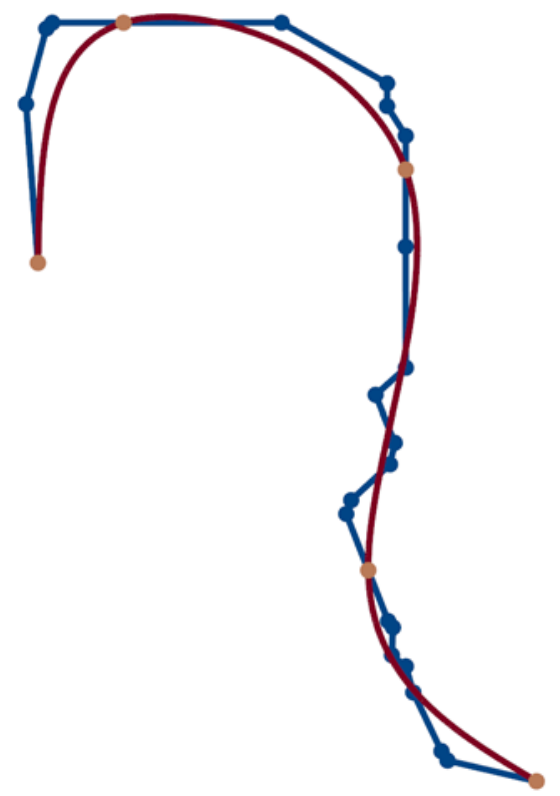

Fig.10. Spline example with five nodes

Another example of approximation for another polyline is shown in Fig. 11 and 12. Here $N$ is equal to 3 and 4 , respectively.

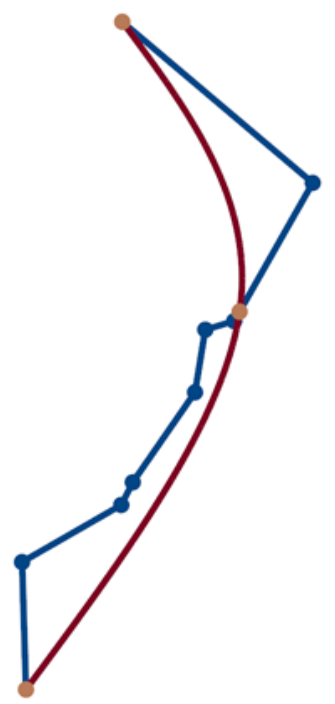

Fig. 11. Spline example with three nodes

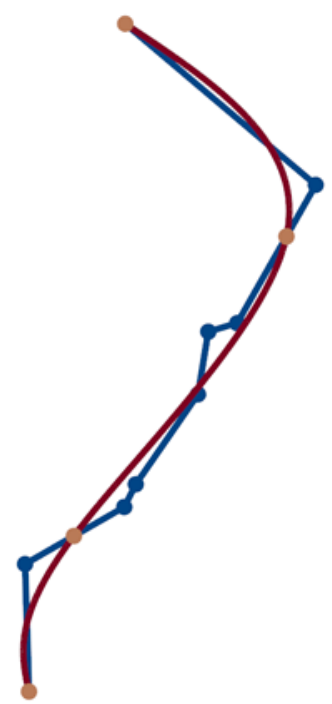

Fig. 12. Spline example with three nodes

Let us construct by the proposed method splines for all polylines representing the paths found on the graph between the inputs and outputs of the fluidic elements. In fig. 13, the polylines are shown in yellow, and the splines that approximate them are shown in crimson. For each spline, we construct the channel boundaries so that the spline passes along the center of the channel, and the channel width varies linearly from the width of the outlet of the fluidic element to the width of the entrance of the receiving fluidic element. Figure 14 
shows the final geometric model of the channels for the considered example of the fluidic device.

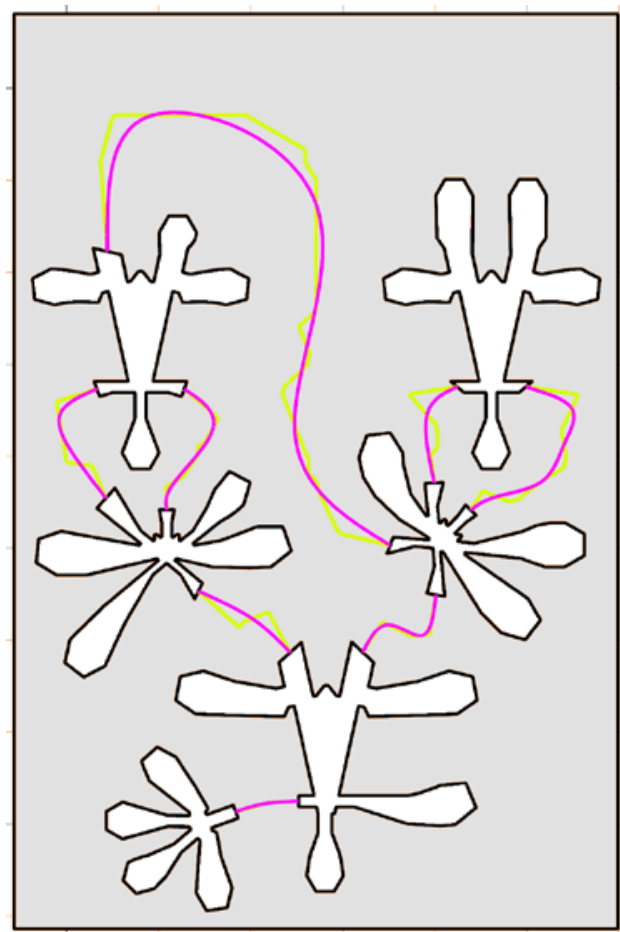

Fig. 13. The result of spline approximation of paths on the graph of a straight skeleton

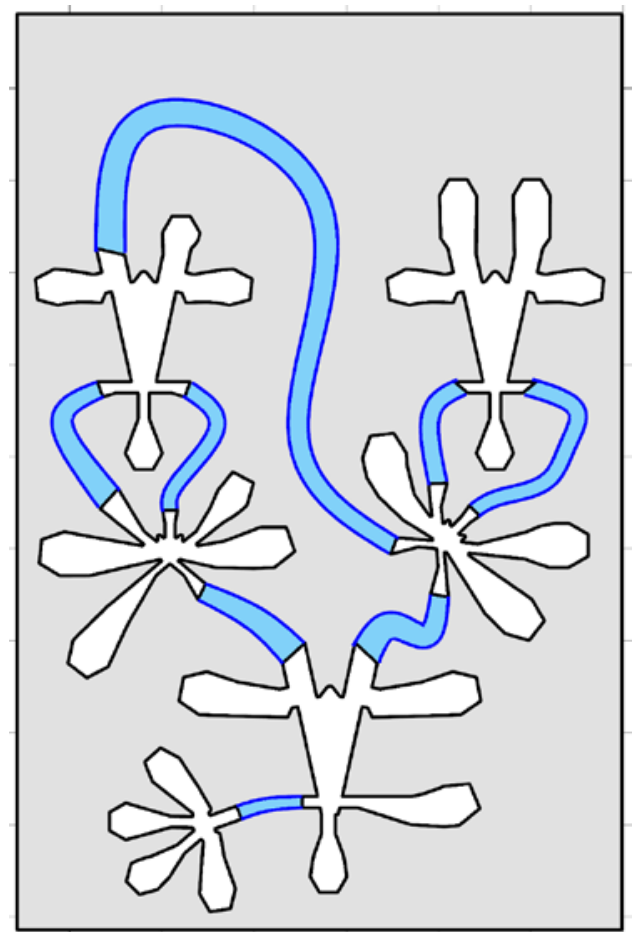

Fig.14. The geometric model of the channels of the fluidic device

\section{Conclusion}

Thus, a method for routing of fluidic circuits is proposed, including the construction of a topological model of channels using skeletons of polygonal figures and the construction of a geometric model of channels using compound splines. A method for approximating polylines by compound splines is proposed. An example of routing of channels of an fluidic device is considered.

\section{References}

1. I.V. Kuzichev, V.A. Romakin, A.V.Balabanov, Komp'yuternaya sistema na osnove 3D-modelej dlya inzhenernogo analiza integral'nyh strujnyh ustrojstv rezervnyh sistem upravleniya letatel'nymi apparatami // Materialy Mezhdunarodnogo molodyozhnogo foruma "Budushchee aviacii i kosmonavtiki za molodoj Rossiej" (Rybinsk, 2015). Rybinsk: Izd-vo RGATU imeni P. A. Solov'yova. S. 52-59 (2015)

2. V. A. Romakin, Routing of fluidic circuits using skeletons of polygonal figures // IOP Conference Series: Materials Science and Engineering. Vol. 971. C. 1-4 Article number 052038 (2020)

3. V. V. Borisenko, Construction of optimal Bezier splines // Fundamentalnaya i prikladnaya matematika, vol. 21, no. 3, pp. 57-72 (2016) 